\title{
Vacunas contra la COVID-19 y aparición de variantes del SARS-CoV-2. Reflexionando sobre el caso de México
}

\section{Vaccines against COVID-19 and the emergence of SARS-CoV-2 variants of concern. Reflections on the case of Mexico}

\author{
José A. Díaz-Quiñónez* \\ División de Estudios de Posgrado, Facultad de Medicina, Universidad Nacional Autónoma de México, Ciudad de México, México
}

Blut ist ein ganz besonderer Saft. Goethe

Consideradas como uno de los inventos más importantes en la historia de la humanidad, las vacunas representan la intervención más simple y rentable para proteger contra epidemias y pandemias. Básicamente, los beneficios se relacionan con la disminución de la mortalidad y la morbilidad, e incluyen, a su vez, beneficios económicos al evitar la hospitalización, prevenir la discapacidad a largo plazo y mantener la economía. Sin embargo, el desarrollo de nuevas vacunas es un proceso muy complejo, dado que deben someterse a procesos extensivos de revisión y aprobación por parte de agencias reguladoras nacionales antes de que puedan ser aplicadas a la población $n^{1,2}$. Los programas nacionales de vacunación también son extremadamente complejos en su diseño, estrategia y ejecución, ya que requieren de una logística detallada y una cadena de suministro segura para llevar las vacunas a los sitios de aplicación ${ }^{3}$.

En condiciones normales, una compañía debe invertir entre 314 y 2,800 millones de dólares (USD de 2018) para obtener una nueva vacuna desde el laboratorio hasta los pacientes ${ }^{4,5}$. Estos montos reflejan en gran medida el costo de los productos que fracasan en los ensayos clínicos y el costo del tiempo invertido en el desarrollo. Además, se requieren entre 10 y 15 años para que una vacuna experimental llegue al mercado y la probabilidad de que sea eventualmente aprobada para uso en humanos se estima en menos del $12 \%$. Las empresas que producen vacunas se encuentran en la vanguardia de la innovación biomédica, por lo que protegen sus productos y procesos mediante políticas sólidas de propiedad intelectual en mercados competitivos que recompensen su inversión ${ }^{8,9}$. Así que las políticas públicas de los países son de capital importancia para el establecimiento y el desarrollo de este sector.

El coronavirus 2 del síndrome respiratorio agudo grave (SARS-CoV-2) apareció en un momento de madurez del conocimiento científico básico en algunas áreas, incluyendo la genómica, la biología estructural, la cartografía antigénica y la vacunología reversa. De esta manera, la investigación científica global pudo realizarse a velocidad pandémica y el desarrollo de vacunas también sufrió un cambio de paradigma $^{10}$. El primer genoma completo del virus fue liberado el 10 de enero de 2020 (a la fecha están depositadas más de 500,000 secuencias en bases de datos públicas y privadas, incluyendo 839 secuencias de individuos en México). Los países de altos ingresos rápidamente crearon capacidad de fabricación, implementaron tecnologías con plataformas novedosas, obtuvieron licencias para fabricar a gran escala, transfirieron tecnologías y adaptaron los procesos de fabricación ${ }^{11,12}$. La realización de ensayos clínicos durante la pandemia por enfermedad por coronavirus 2019 (COVID-19) plantea desafíos adicionales, incluida la forma en que se realizan y cómo suceden los procesos de aprobación. Así, el primer candidato a vacuna entró en ensayos clínicos el 16 de marzo de 2020 en
Correspondencia:

0016-3813/@ 2021 Academia Nacional de Medicina de México, A.C. Publicado por Permanyer. Este es un artículo open access bajo la licencia
*José A. Díaz-Quiñonez

E-mail: adiazq@unam.mx

Fecha de recepción: 17-03-2021

Fecha de aceptación: 18-03-2021 CC BY-NC-ND (http://creativecommons.org/licenses/by-nc-nd/4.0/)
Gac Med Mex. 2021;157:125-126

Disponible en PubMed

www.gacetamedicademexico.com 
EE.UU. y, a día de hoy, existe una docena de vacunas autorizadas para uso humano en 64 países, con más de 5,000 ensayos clínicos registrados en todo el mundo ${ }^{13,14}$. En medio del paradigma pandémico, esta exitosa experiencia de colaboración global para la obtención de nuevas vacunas (con atributos de seguridad y eficacia, en tiempos tan reducidos) ha renovado la discusión respecto de un sistema global de financiamiento, vigilancia y asignación de vacunas ${ }^{15}$. En México nos debe llevar a una profunda reflexión respecto de la implementación de políticas públicas intersectoriales en salud, propiedad intelectual, ciencia, tecnología e innovación. De nuevo, tenemos una oportunidad para establecer sistemas de preparación y respuesta ante pandemias y emergencias en salud que, sin duda, seguirán apareciendo en el futuro.

Las vacunas desarrolladas contra el SARS-CoV-2 dependen en gran medida de la inducción de anticuerpos que son capaces de neutralizarlo. El surgimiento de linajes del virus con capacidad para escapar de las respuestas inmunitarias previamente montadas, tanto en la vacunación como en la inmunidad adquirida por infección natural, es de especial interés para la salud pública $^{16,17}$. La circulación de estas variantes de interés pone en riesgo la eficacia de las vacunas desarrolladas y la protección de las poblaciones vacunadas. Aunque varios grupos en el mundo están trabajando ya para modificar las vacunas, actualmente no sabemos si las tres variantes de interés identificadas en México (B.1.1.7, P.1 y CAL.20C), y posiblemente otras, representan un riesgo para las vacunas autorizadas (Oxford-AstraZeneca, Pfizer-BioNtech, Sputnik V, CanSino y SinoVac, a 9 de marzo de 2021). Para ello se requiere una estrecha caracterización viral (por medio de novedosas tecnologías de secuenciación, análisis bioinformático y epidemiología genómica) de los linajes circulando en nuestro país. Dado que la disponibilidad de las vacunas es solo una primera parte de la ecuación, la estrategia para alcanzar coberturas que disminuyan la transmisión (con ello la posibilidad de aparición de nuevas variantes) y la implementación de una vigilancia genómica intensiva con investigaciones epidemiológicas bien coordinadas (de naturaleza intersecretarial) son retos urgentes para la salud pública mexicana.

\section{Bibliografía}

1. Plotkin S, Robinson JM, Cunningham G, Iqbal R, Larsen S. The complexity and cost of vaccine manufacturing - An overview. Vaccine. 2017;35(33):4064-71.

2. Xue QC, Ouellette LL. Innovation policy and the market for vaccines. J Law Biosci. 2020;7(1):Isaa026.

3. World Health Organization. Guidance on developing a national deployment and vaccination plan for COVID-19 vaccines [Internet]. World Health Organization. Disponible en: https://www.who.int/publications/i/item/ WHO-2019-nCoV-Vaccine_deployment-2020.1

4. Gouglas D, Thanh Le T, Henderson K, Kaloudis A, Danielsen T, Hammersland NC, et al. Estimating the cost of vaccine development against epidemic infectious diseases: a cost minimisation study. Lancet Global Health. 2018;6(12):e1386-e1396.

5. Wouters OJ, McKee M, Luyten J. Estimated research and development investment needed to bring a new medicine to market, 2009-2018. JAMA. 2020;323(9):844-53.

6. Carter PH, Berndt ER, DiMasi JA, Trusheim M. Investigating investment in biopharmaceutical R\&D. Nat Rev Drug Discov. 2016;15(10):673-4.

7. DiMasi JA, Grabowski HG. The cost of biopharmaceutical R\&D: Is biotech different? Manage Decis Econ. 2007;28:469-79.

8. Grabowski HG, DiMasi JA, Long G. The roles of patents and research and development incentives in biopharmaceutical innovation. Health Aff (Millwood). 2015;34(2):302-10.

9. Chit A, Grootendorst P. The effect of government policy on pharmaceutical innovation [Internet]. Oxford Research Encyclopedias: Economics and Finance; 2019. Disponible en: https://doi.org/10.1093/ acrefore/9780190625979.013.77

10. Lurie N, Saville M, Hatchett R, Halton J. Developing Covid-19 vaccines at pandemic speed. N Engl J Med. 2020;382(21):1969-73.

11. Jeyanathan M, Afkhami S, Smaill F, Miller MS, Lichty BD, Xing Z. Immunological considerations for COVID-19 vaccine strategies. Nat Rev Immunol. 2020;20(10):615-32.

12. Singh R, Kang A, Luo X, Jeyanathan M, Gillgrass A, Afkhami S, Xing Z. COVID-19: Current knowledge in clinical features, immunological responses, and vaccine development. FASEB J. 2021;35(3):e21409.

13. World Health Organization. Draft landscape of COVID-19 candidate vaccines [Internet]. World Health Organization [acceso: 8 de marzo de 2021]. Disponible en: https://www.who.int/publications $/ \mathrm{m} /$ item/ draft-landscape-of-covid-19-candidate-vaccines 2020

14. Johns Hopkins University of Medicine. Coronavirus Resource Center. Vaccines [Internet]. Johns Hopkins University of Medicine [acceso: 8 de marzo de 2021]. Disponible en: https://coronavirus.jhu.edu/vaccines

15. Mascola JR, Graham BS, Fauci AS. SARS-CoV-2 viral variants-tackling a moving target. JAMA. 2021 Feb 11. doi: 10.1001/jama.2021.2088. Online ahead of print.

16. Wang $P$, Nair MS, Liu L, Iketani S, Luo Y, Guo Y, et al. Antibody resistance of SARS-CoV-2 variants B.1.351 and B.1.1.7. Nature. 2021 Mar 8. doi: 10.1038/s41586-021-03398-2. Online ahead of print

17. McCarthy KR, Rennick LJ, Nambulli S, Robinson-McCarthy LR, Bain WG, Haidar G, et al. Recurrent deletions in the SARS-CoV-2 spike glycoprotein drive antibody escape. Science. 2021;371(6534):1139-42. 